\section{An observational study of laterality errors in a sample of clinical records}

\begin{abstract}
Background Confusing left with right eyes can have a potentially serious adverse outcome. The most extreme occurrence is wrong site surgery but even potentially less serious errors can undermine patient confidence in their medical care. This study was designed to look into how often this could be detected in clinical notes.

Methods An observational study conducted in an ophthalmic hospital. Hundred patients were randomly selected and their clinical notes retrieved. Notes were analysed for the number of left/right transpositions, which part of the notes they were found and whether they were corrected.
\end{abstract}

Results Forty-four transposition errors were found in 32 sets on notes. The commonest error was drawing the eye on the wrong side of the page. The commonest place where errors were found was in the written outpatient notes. Nineteen of the errors had evidence of later correction. Three consent forms had the incorrect eye denoted and one patient was

Department of Ophthalmology, Sunderland Eye Infirmary, Sunderland, UK

Correspondence: SG Fraser, Department of Ophthalmology,

Sunderland Eye Infirmary, Queen Alexandra Road, Sunderland SR2 9HP, UK Tel: + 4401915699 853; Fax: + 4401915699060 . E-mail: s.g.fraser@ncl.ac.uk

Received: 9 May 2006 Accepted in revised form: 9 August 2006

Published online:

15 September 2006

Conflict of interest: None declared listed for surgery on the wrong side although this error was corrected before the operation. Conclusion As far as we are aware, this study is the first to look at how often, in standard clinical notes, left/right transposition occurs. Although a direct link cannot made between their occurrence and later wrong side surgery, intuitively it would be reasonable to think it could increase the likelihood if other defences were to fail. We make a number of recommendations that might reduce this confusion and therefore more serious consequences.

Eye (2008) 22, 340-343; doi:10.1038/sj.eye.6702590; published online 15 September 2006

Keywords: laterality; ophthalmology; transposition errors; wrong site surgery; patient safety
I EIGhrably and SG Fraser

\section{Introduction}

Performing a procedure on the wrong side of the body is a devastating complication for patient and practitioner. It is a particular spectre for those who work on paired organs such as kidneys, limbs, ears, lungs, ovaries, or eyes. ${ }^{1}$ Traquair $^{2}$ described in 1947 'the feared complication of wrong side enucleations'. Possibly less seriously - but caused by the same faulty processes, wrong side medication (eg wrong eye drop, injection into the wrong joint) also has the potential for harm.

Fortunately, wrong side procedures are rare-especially if there are systems in place to try to prevent them. ${ }^{3}$ It is well recognized that, like many medical errors, wrong side surgery usually occurs not because of a single catastrophic error but as a result of a series of small errors. These apparently trivial mistakes may occur anywhere in the patient's journey from the initial referral letter to the operating room.

One such mistake is accidental transposition of left and right. Confusing left with right is a common occurrence in everyday life and there seems no reason why this should be less so in medical practice-despite the potentially more serious consequences. At one extreme it may result in the wrong eye being operated upon, but more likely it is inconsequential - either because other defences are in place or the error occurs in a situation where harm is unlikely. It is possible, however, that occasionally these seemingly unimportant errors, could be the precursors of a more serious error (as well as undermining patient confidence with the practitioner or hospital). The sequence of events for wrong site surgery has to begin somewhere and it may be that the more often the systems in place to avoid procedures on the wrong side are stressed by these small errors, the more likely they are to eventually fail. 
The purpose of this study was therefore to assess how often the small, but potentially serious error, of mixing left and right eyes occurred within a series of clinical notes. A number of authorities have suggested reasons that wrong side surgery happens but, as far as we are aware there is no literature that has looked at the number of times left right confusion occurs. Although it is unlikely that any of these occurrences would in themselves lead to wrong site procedures they may represent a measure of how easily and how often these transposition errors can happen.

\section{Methods}

The study was conducted wholly at Sunderland Eye Infirmary, which is a dedicated ophthalmic hospital with its own on-site medical records. Using the computerized patient database, 100 sets of patient's notes (which fulfilled the criteria below) were randomly selected (using a random number generator) and retrieved. As each set of notes contained a huge amount of information, the most recent visit was identified and the previous 12 (arbitrarily decided) consecutive visits were analysed. Patients were excluded if they did not have 12 visits or had nonconsecutive visits (eg were discharged and rereferred or had a number of nonattendances).

For each set of notes, the 12 visits were analysed for:

- The number of occasions when sides were transposed, i.e., left and right were confused.

- Which part of the notes it occurred, for example, written notes, clinic letters, prescriptions, consent forms, operative notes.

- Nature of the transposition, for example, clinical drawing wrong side of page, eye drops prescribed for left eye rather than right.

- Whether there was evidence that the error was corrected, for example, amended at the time or at a later date or mentioned in a later note or letter as having been incorrect.

Errors that occurred at the same visit and were related were not counted twice, for example, if an error was made in the written notes and this led to the same error in the clinic letter, this was counted as one error and not two. However, if at the next or subsequent visit the same transposition error occurred this was counted as a new error.

\section{Results}

Of the 100 randomly chosen notes, 32 had at least one left/right transposition error. Nine sets had two errors, whereas one set had four errors. This meant that, in total, there were 44 transposition errors. When the errors were analysed it seemed logical to group them as shown:

\section{Type of transcription error}

- Drawing wrong side page (conventionally in ophthalmology, handwritten patients notes have the eyes drawn as seen by the practitioner, that is, the left eye is drawn on the right-hand side of the page and vice versa for the right. Note that in the unit this study was undertaken, the written patient records do not have a line down the middle of the page separating left from right as some departments do).

- Diagnosis denoted for incorrect eye (in unilateral diseases).

- Treatment denoted wrong side.

- Consent for procedure forms.

- Listed for surgery.

- Miscellaneous, for example, visual acuity.

\section{Where the transposition occurred}

- In the written outpatient notes.

- Letters to the patient's primary care physician.

- In the theatre notes or operation sheet.

- On the consent form.

\section{Was the transposition corrected?}

Either at the time (the incorrect side was crossed out and replaced), at a later time (with a date) or in a later entry in the notes. It was of course only possible with the latter two to know when any correction occurred.

The results from each section are presented in Table 1.

\section{Discussion}

Our results suggest a surprisingly high rate of transposition errors with 44 in 32 sets of notes. The commonest type of transposition occurred when the eye was drawn on the wrong side of the page. This occurred equally in the surgical notes (ie description of the operation) as in the outpatient notes. This error was corrected on two occasions.

The second commonest error was when treatment was written down for one eye when it should have been the other. This was mainly when it was written in the notes and only three outpatient letters were wrong - this suggests that this is an error of transcription, that is, the side was written incorrectly but the transcriber did know the correct side. This conclusion is strengthened by the fact that most of these errors were corrected. We have no evidence that any of these errors led to a patient getting the wrong medication - although we have previously found quite a high rate of prescription errors in the ophthalmic outpatient setting. ${ }^{4}$ 
Table 1 Number of times transposition errors were found within the notes, the place the error was found, and the number that were corrected

\begin{tabular}{lcccrc}
\hline & Total number occurrences & Surgical notes & Letters & Outpatient notes & Corrected \\
\hline Drawing wrong side page & 15 & 7 & 0 & 8 & 1 \\
Diagnosis denoted for wrong side & 4 & 1 & 2 & 3 & 3 \\
Treatment denoted for wrong side & 13 & 0 & 3 & 0 & 9 \\
Consent for surgery & 3 & 3 & 0 & 0 & 0 \\
Listed for wrong side surgery & 1 & 0 & 0 & 8 & 1 \\
Miscellaneous & 8 & 0 & 0 & 29 & 19 \\
Total & 44 & 11 & 5 & & 0 \\
\hline
\end{tabular}

Four patients had the side of their diagnosis mixed up - but three of these were corrected. Three consent forms had the incorrect eye written on them, but none of these were corrected - although all of these patients had the correct eye procedure. Of relevance is the fact that all three were outpatient procedures (eg laser or minor operations) and because of this the consent verification systems were probably less robust.

One patient was listed for an operation on the incorrect eye and this was not picked up until the day of surgery. This error persisted through a number of checks (the process began when the wrong eye was written in the outpatient notes) and was not corrected until the patient was consented shortly before the operation.

Although in theory any transposition error could lead to wrong side procedure or treatment, intuitively the nearer to the procedure the transposition occurs the more likely that the wrong eye could be operated upon.

As discussed previously the concept of wrong side surgery is far from new. ${ }^{2}$ With the increased interest in the study of patient safety over the past decade, a number of groups have looked at some of the reasons for this error. Not surprisingly, there can be a number of causes - from lack of a standard marking system, difficult patient anatomy, lack of patient/carer involvement to time pressures on large lists ${ }^{5}$ as well as possible solutions. ${ }^{3}$ Of potential importance, the UK National Patient Safety Agency has produced a standardized marking and verification system that if adopted nationally could have a major effect on reducing wrong site surgery (http://www.npsa.nhs.uk/site/ media/documents/885_CSS\%20PSA06\%20FINAL.pdf).

We are not aware of a study such as ours that has looked at the number of times laterality is confused in clinical notes. It is of course difficult to ascertain a direct link between these apparently insignificant errors and the serious error of operating on the wrong eye, but it seems reasonable to suggest that there may be a link. This could either be because a more serious error has to begin somewhere or because the more time the defences are hit (ie the more often the sides are mixed up in the notes) the more likely that these defences will eventually be breached. It may be by reducing the occurrence of these everyday transposition errors eventually a patient will be saved from a wrong side procedure.

There are a number of potential flaws in the paper that may have had an effect on our findings. Although we counted the same transposition error on different visits it is possible that the practitioner was sometimes influenced by this previous mistake, that is, simply copying the previous diagram or treatment regime. This would tend to overestimate the total number of errors - although the principle that one error is more likely to lead to a subsequent one would not be affected by this.

The study was of course retrospective which inevitably degrades the data. This is especially so for the data regarding correction of the error when it was not possible to know when the correction occurred. Our notes retrieval was $100 \%$ for suitable patients (ie 12 consecutive visits) reducing the bias of selective notes retrieval.

\section{Recommendations}

Our study suggests some strategies that may reduce the likelihood of left/right transpositions. Although specifically taken from ophthalmic notes, perhaps some of the recommendations may be universal:

(1) The system of drawing the clinical findings as the patient has been seen is a very natural way of describing examination findings, it does, however, seems to contain possible ways of mixing up the eyes. Possible improvements:

i The use printed pages with a central margin separating left from right with all findings only drawn on the appropriate side.

ii To further improve precision, left and right eyes could be printed at the top of each page.

(2) Related to this, treatments should be written only on the 'correct' side of the page. This is especially so if the patient is putting different medications in their eyes. This could even be extended to bilateral treatment, when each eye treatment should be 
written separately. Although time consuming, this should improve precision.

(3) Avoid using the abbreviations for left and right-L and R. Too often - especially when written quickly - they can be mixed up.

(4) With the increasing use of electronic records left/ right transposition errors may reduce - although data is only as accurate as its input.

(5) Involvement of the patient and or relatives remains very important. ${ }^{6}$ When consenting patients for surgery or prescribing their drops, explaining to the patient what is to be done and listening to any queries remains an important mechanism in reducing laterality errors.

In conclusion, this simple observational study looked at 100 sets of clinical notes and found 32 that had one or more errors making a total of 44 transposition errors. Although none of these resulted in patient harm each one could have been the starting point for a series of errors that, if other defences failed, could have resulted in wrong site surgery.

We have made a number of recommendations to reduce these transposition errors, and although these may reduce the number of this type of errors, we suggest that there should be a continued and concerted effort to produce robust systems to further reduce the likelihood of this type of simple mistake that could be the precursor of a more catastrophic error.

\section{References}

1 Gibbs VC. Patient safety practices in the operating room: correct-site surgery and nothing left behind. Surg Clin N Am 2005; 85(6): 1307-1319, xiii.

2 Traquair H. Removal of the wrong eye. Br J Ophthalmol 1947; 31: 8-12.

3 Fraser S, Adams W. Wrong site surgery-a preventable complication? Br J Ophthalmol 2006; 90: 814-816.

4 Mandal K, Fraser SG. The incidence of prescribing errors in an eye hospital. BMC Ophthalmol 2005; 5(1): 4.

5 Saufl NM. Universal protocol for preventing wrong site, wrong procedure, wrong person surgery. J Perianesth Nurs 2004; 19(5): 348-351.

6 DiGiovanni CW, Kang L, Manuel J. Patient compliance in avoiding wrong-site surgery. J Bone Joint Surg Am 2003; 85-A(5): 815-819. 